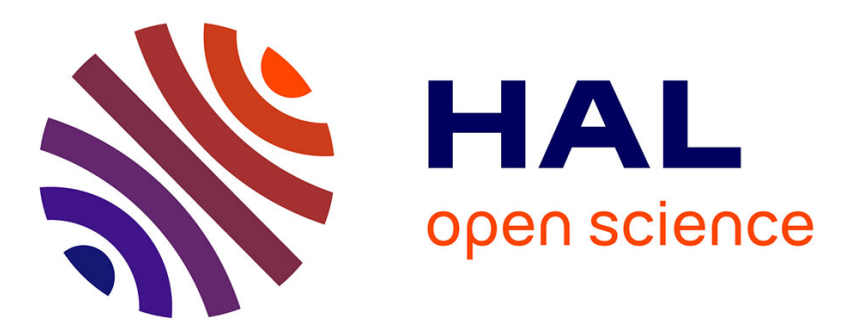

\title{
Numerical simulation of large amplitude oscillatory shear of a high-density polyethylene melt using the MSF model
}

P. Wapperom, Adrien Leygue, Roland Keunings

\section{- To cite this version:}

P. Wapperom, Adrien Leygue, Roland Keunings. Numerical simulation of large amplitude oscillatory shear of a high-density polyethylene melt using the MSF model. Journal of Non-Newtonian Fluid Mechanics, 2005, 130 (2-3), pp.63-76. 10.1016/j.jnnfm.2005.08.002 . hal-01007132

\section{HAL Id: hal-01007132 \\ https://hal.science/hal-01007132}

Submitted on 20 Nov 2017

HAL is a multi-disciplinary open access archive for the deposit and dissemination of scientific research documents, whether they are published or not. The documents may come from teaching and research institutions in France or abroad, or from public or private research centers.
L'archive ouverte pluridisciplinaire HAL, est destinée au dépôt et à la diffusion de documents scientifiques de niveau recherche, publiés ou non, émanant des établissements d'enseignement et de recherche français ou étrangers, des laboratoires publics ou privés. 


\title{
Numerical simulation of large amplitude oscillatory shear of a high-density polyethylene melt using the MSF model
}

\author{
P. Wapperom ${ }^{\mathrm{a}, *}$, A. Leygue ${ }^{\mathrm{b}}$, R. Keunings ${ }^{\mathrm{b}}$ \\ a Department of Mathematics, Virginia Tech, Blacksburg, VA 24061, USA \\ ${ }^{\mathrm{b}}$ CESAME, Université catholique de Louvain, B-1348 Louvain-la-Neuve, Belgium
}

\begin{abstract}
We study the flow response in large amplitude oscillatory shear of the molecular stress function (MSF) model that has recently been proposed by Wagner et al. [M.H. Wagner, P. Rubio, H. Bastian, The molecular stress function model for polydisperse polymer melts with dissipative convective constraint release, J. Rheol. 45 (2001) 1387-1412]. The MSF model is derived from molecular theory and has only two parameters to describe the non-linear material response. The model predictions are analysed in both the frequency and time domain. It shows good agreement with experimental data for a linear high-density polyethylene melt. At low and medium strains, MSF model predictions are in excellent agreement with experimental data and predictions of a six-mode Giesekus model which has six parameters to describe the non-linear material response. At medium strains, the basic Doi-Edwards model, which has no non-linear parameters, already underpredicts the data. At high strains, the MSF model predictions agree slightly better with the experimental data than the Giesekus model. Surprisingly, however, it is the Doi-Edwards model that shows excellent agreement with experimental data at high strains. For the linear melt we consider, it outperforms the models that have non-linear parameters, both in the time and frequency domain.
\end{abstract}

Keywords: LAOS; Integral MSF model; Deformation field method; Linear polymer melts

\section{Introduction}

Molecular models have become increasingly popular to describe the complex rheological behavior of entangled polymeric liquids. Practically all recently developed molecular models are extensions of the Doi-Edwards theory. Although the basic DoiEdwards (DE) model [1] can successfully predict the damping function and the plateau modulus of linear viscosity, it has some important deficiencies like excessive shear thinning in fast shearing flows. Recent extensions of the Doi-Edwards theory have alleviated these shortcomings. We consider one of such extensions, the molecular stress function (MSF) model which has recently been proposed by Wagner et al. [2]. The non-linear response is captured through the introduction of an extra evolution equation for the molecular stress function. The model has two additional material parameters, one to describe extensional and one to describe shear flows. With only two non-linear parame-

\footnotetext{
* Corresponding author.

E-mail address: wapperom@math.vt.edu (P. Wapperom).
}

ters, the MSF model is able to accurately predict the non-linear response in start up of shear and extension for a commercial linear high-density and branched low-density polyethylene [2].

The linear spectrum of polymer melts is normally determined in small amplitude oscillatory shear flow. At low strains, the material response is approximately linear. At larger strains, however, non-linear effects do play an important role. Early large amplitude oscillatory shear (LAOS) experiments date already from almost 50 years ago [3]. A recent overview of LAOS has been given by Giacomin and Dealy [4]. Wilhelm et al. [5,6] introduced high-sensitivity Fourier-transform rheology and analysed large amplitude step shear oscillations of polymer melts [7]. Very recently, Debbaut and Burhin [8] performed LAOS experiments on a commercial high-density polyethylene melt up to high strains of 10 . Their simulations with a Giesekus model showed good agreement for moderate non-linear regimes and larger deviations for the most non-linear regimes that were experimentally achievable.

In this paper, we study the rheometrical response of the MSF model in large amplitude oscillatory shear flow. In particular, we investigate whether such models derived from molecular theory 
are able to accurately predict experimental data of a commercial linear polymer melt. In order to perform the simulations in an efficient and accurate manner, we modify the deformation field method of Hulsen et al. [9] (Section 4). After validation of the numerical technique in Section 6, we show that the MSF model, having only one relevant material parameter in LAOS, can accurately predict experimental results for a linear highdensity polyethylene melt [8]. At medium strains, the performance is comparable to that of a six-mode Giesekus fluid having six material parameters to describe the non-linear regime. At these strains the basic Doi-Edwards model already underpredicts the experimental data. At the higher strains, both the MSF and Giesekus model overpredict the experimental data, although the MSF model renders slightly to significantly better predictions depending on the value of the non-linear material parameter. Completely unexpectedly, it is the Doi-Edwards model that shows excellent agreement with the experimental data at the high strains. Then, the DE model, having no non-linear material parameters, outperforms both non-linear models.

\section{Governing equations}

For the MSF model the stress is related to the deformation history by

$\boldsymbol{T}(t)=5 \int_{-\infty}^{t} m\left(t-t^{\prime}\right) f_{t^{\prime}}^{2}(t) \boldsymbol{Q}\left[\boldsymbol{B}_{t^{\prime}}(t)\right] \mathrm{d} t^{\prime}$,

where $\boldsymbol{B}_{t^{\prime}}(t)$ is the Finger tensor which measures the deformation of a fluid particle at the current time $t$ with respect to a reference time $t^{\prime}$. The memory function $m$ assumes the classical multimode Maxwell form

$m\left(t-t^{\prime}\right)=\sum_{i} \frac{G_{i}}{\lambda_{i}} \int_{-\infty}^{t} \mathrm{e}^{-\left(t-t^{\prime}\right) / \lambda_{i}}$,

where $G_{i}$ are the moduli and $\lambda_{i}$ are the relaxation times of the fluid.

The tensor $\boldsymbol{Q}$ is the strain measure for which the independent alignment approximation was used in the original derivation in [2]. Instead, we use the Currie approximation to the DoiEdwards deformation tensor [10]. The orientation tensor $\boldsymbol{Q}$ is then directly related to the Finger strain $\boldsymbol{B}_{t^{\prime}}$ and the Cauchy strain $\boldsymbol{B}_{t^{\prime}}^{-1}$ by

$\boldsymbol{Q}=\frac{1}{J-1} \boldsymbol{B}_{t^{\prime}}-\frac{1}{(J-1)\left(I_{2}+3.25\right)^{1 / 2}} \boldsymbol{B}_{t^{\prime}}^{-1}$.

Here, $J=I_{1}+2\left(I_{2}+3.25\right)^{1 / 2}$ and $I_{1}$ and $I_{2}$ are the first and second invariants of $\boldsymbol{B}_{t^{\prime}}$, respectively. For large amplitude oscillatory shear flow this is a very good approximation to the Doi-Edwards tensor using the independent alignment approximation, as we show in Section 7.

The MSF model is completed by an evolution equation for the Finger tensor and the molecular stress function $f_{t^{\prime}}$. The Finger tensor is governed by

$\frac{\mathrm{D} \boldsymbol{B}_{t^{\prime}}}{\mathrm{D} t}=\boldsymbol{\kappa} \cdot \boldsymbol{B}_{t^{\prime}}+\boldsymbol{B}_{t^{\prime}} \cdot \boldsymbol{\kappa}^{\mathrm{T}}$, where $\boldsymbol{\kappa}$ is the transpose of the velocity gradient. For the MSF model, the evolution equation for the square of the molecular stress function takes different forms for linear and branched polymers. For linear polymers, we have

$\frac{\mathrm{D} f_{t^{\prime}}^{2}}{\mathrm{D} t}=f_{t^{\prime}}^{2}\left[\boldsymbol{\kappa}: \boldsymbol{Q}-\frac{1}{f_{t^{\prime}}^{2}-1} \mathrm{CR}\right]$

while for branched polymers the right-hand side is slightly modified $[2,11]$. The dissipative constraint release CR is expressed as

$$
\mathrm{CR}=\frac{1}{2}\left(f_{t^{\prime}}^{2}-1\right)^{2}\left[a_{1} \sqrt{\boldsymbol{A}_{1}^{2}: \boldsymbol{Q}}+a_{2} \sqrt{\left|\boldsymbol{A}_{2}: \boldsymbol{Q}-\boldsymbol{A}_{1}^{2}: \boldsymbol{Q}\right|}\right]
$$

where $\boldsymbol{A}_{1}^{2}$ and $\boldsymbol{A}_{2}$ are second-order Rivlin-Erickson tensors which are related to the rate-of-deformation tensor $\boldsymbol{d}=(\boldsymbol{\kappa}+$ $\left.\boldsymbol{\kappa}^{\mathrm{T}}\right) / 2$ and rate-of-rotation tensor $\boldsymbol{w}=\left(\boldsymbol{\kappa}-\boldsymbol{\kappa}^{\mathrm{T}}\right) / 2$ by

$\boldsymbol{A}_{1}^{2}=4 \boldsymbol{d}^{2}, \quad \boldsymbol{A}_{2}=\frac{\mathrm{D} \boldsymbol{A}_{1}}{\mathrm{D} t}+\boldsymbol{A}_{1}^{2}+2 \boldsymbol{w} \cdot \boldsymbol{d}+2 \boldsymbol{d} \cdot \boldsymbol{w}^{\mathrm{T}}$.

The parameters $a_{1}$ and $a_{2}$ in Eq. (6) are the only two non-linear parameters in the MSF model. The only parameter that is relevant in steady non-rotational flows is $a_{1}$, since $\boldsymbol{A}_{2}: \boldsymbol{Q}-\boldsymbol{A}_{1}^{2}: \boldsymbol{Q}$ vanishes in that case. The value of $a_{1}$ can be determined by fitting extensional flow data. Next, $a_{2}$ can be obtained from shear viscosity and first normal stress data.

The MSF theory is an extension of the basic Doi-Edwards tube theory [1]. The difference between the models is the inclusion of the molecular stress function for the MSF model. To evaluate the improvement of this model in large amplitude oscillatory shear, we use the Doi-Edwards model for comparison. The governing equations are then Eqs. (1)-(4) with $f_{t^{\prime}}^{2}=1$ in the expression for the polymer stress. The DE model has no non-linear parameters and is fully characterized by the linear spectrum.

\section{Large amplitude oscillatory shear flow}

In large amplitude oscillatory shear flow a fluid is subject to a periodic shear deformation $\gamma$ with amplitude $\gamma_{0}$ and frequency $v$,

$\gamma(t)=\gamma_{0} \sin (2 \pi v t)$

The deformation is applied for $t>0$ and up to $t=0$ the fluid is assumed at rest. The corresponding periodic shear rate $\dot{\gamma}$ equals

$\dot{\gamma}(t)=2 \pi \nu \gamma_{0} \cos (2 \pi \nu t)$.

With this shear rate, the polymeric stress is obtained as a function of time from the constitutive equations (1)-(4) and the evolution equation for the molecular stress function for linear polymers, Eqs. (5)-(7). Since the stress response attains a steady periodic state, a Fourier transform facilitates a detailed quantitative analysis. The shear stress is decomposed into an infinite sum of 
trigonometric functions,

$T_{x y}(t)=\frac{A_{0}}{2}+\sum_{n=1}^{\infty} A_{n} \sin (2 n \pi v t)+\sum_{n=1}^{\infty} B_{n} \cos (2 n \pi v t)$,

where $A_{n}$ and $B_{n}$ are the Fourier coefficients. For a real signal, these coefficients are given by

$A_{n}=2 v \int_{c}^{c+(1 / v)} T_{x y} \sin (2 n \pi v t) \mathrm{d} t$,
$B_{n}=2 v \int_{c}^{c+(1 / v)} T_{x y} \cos (2 n \pi v t) \mathrm{d} t$

for an arbitrary period ranging from $c$ to $c+(1 / v)$. The Fourier coefficient $\boldsymbol{A}_{0}$ vanishes in view of the two-fold symmetry of the periodic shear stress signal. Standard numerical integration has been used to evaluate the integrals in Eq. (11).

Recently, Debbaut and Burhin [8] have described a new viscometric device to characterize polymer melts in large amplitude oscillatory shear flows. The experimental equipment consisted of an oscillatory device with a closed chamber to allow for higher frequencies. At a temperature of $170^{\circ} \mathrm{C}$, LAOS experiments have been performed at various frequencies and amplitudes for the commercially available high-density polyethylene melt Finathene ${ }^{\circledR} 3802$ YCF. Experimental data are available for the frequencies $v=0.1,0.3,1$, and $3 \mathrm{~Hz}$ and for the amplitudes $\gamma_{0}=0.5,1,2.5,5$, and 10. For the higher frequencies, not all amplitudes can be achieved experimentally. For $v=1 \mathrm{~Hz}$, the highest achievable amplitude is $\gamma_{0}=5$, while for $v=3 \mathrm{~Hz}$ this is $\gamma_{0}=1$. For further reference, we note that the maximum shear rates that occur at the highest amplitude for each frequency are $\dot{\gamma}_{\max } \approx 6.28 \mathrm{~s}^{-1}$ for $v=0.1 \mathrm{~Hz}, \dot{\gamma}_{\max } \approx$ $18.85 \mathrm{~s}^{-1}$ for $v=0.3$ and $3 \mathrm{~Hz}$, and $\dot{\gamma}_{\max } \approx 31.42 \mathrm{~s}^{-1}$ for $v=1 \mathrm{~Hz}$.

\section{Numerical method for simulation in LAOS}

The evolution equations for the Finger tensor $\boldsymbol{B}_{t^{\prime}}$ are easily integrated analytically for large amplitude oscillatory shear, i.e. for the shear rate specified by Eq. (9). We obtain for the nonconstant components of the Finger tensor

$$
\begin{aligned}
& B_{t^{\prime}}^{x y}(t)= \begin{cases}\gamma_{0} \sin (2 \pi v t) & t^{\prime} \leq 0 \\
-\gamma_{0} \sin \left(2 \pi v t^{\prime}\right)+\gamma_{0} \sin (2 \pi v t) & t^{\prime}>0\end{cases} \\
& B_{t^{\prime}}^{x x}(t)=1+\left(B_{t^{\prime}}^{x y}\right)^{2}(t)
\end{aligned}
$$

The strain measure $\boldsymbol{Q}$ can thus be obtained analytically and the only evolution equation that remains to be solved numerically in LAOS is the evolution equation for $f_{t^{\prime}}^{2}$, Eq. (5) for linear polymers. To solve the evolution equation for $f_{t^{\prime}}^{2}$, we follow the idea of Hulsen et al. [9], who used the age $\tau=t-t^{\prime}$ as independent variable instead of $t^{\prime}$ to solve the evolution equation for the Finger tensor $\boldsymbol{B}(t, \tau)=\boldsymbol{B}_{t^{\prime}}(t)$. The introduction of $\tau$ as an independent variable modifies the time derivative in the evolution equation, but leaves the right-hand side unaltered. The proper equation for $f^{2}(t, \tau)=f_{t^{\prime}}^{2}(t)$ involves a derivative with respect to $t$ and $\tau$ resulting from the material derivative while leaving the right-hand side of an evolution equation unaltered. For the molecular stress function describing linear polymers, Eq. (5), we obtain

$\frac{\mathrm{D} f^{2}}{\mathrm{D} t}+\frac{\partial f^{2}}{\partial \tau}=f^{2}\left[\boldsymbol{\kappa}: \boldsymbol{Q}-\frac{1}{f^{2}-1} \mathrm{CR}\right]$

subject to the boundary condition $f^{2}(t, 0)=1$ and the initial condition $f^{2}(0, \tau)=1$, since the fluid is assumed to be at rest for $t<0$.

In [9], the discontinuous Galerkin (DG) method is used for the discretization of the differential equation for the Finger tensor in the $\tau$ direction. For transient flow problems involving large and small time scales, the DG discretization in $\tau$ is not very appealing. Large relaxation times imply a long time before a steady periodic state has been reached. For the simulations in Section 7 we used $200 \mathrm{~s}$. The small relaxation times on the other hand require a fine discretization for small $\tau$. The DG method requires a time step of $\Delta t<\Delta \tau / 6$ for reasons of stability. For our simulations in Section 7, we use $1520 \tau$ intervals which have a minimum length of $9.69 \times 10^{-6} \mathrm{~s}$. For the discontinuous Galerkin method to remain stable, the maximum time step is approximately $1.6 \times 10^{-6}$. To avoid very long computation times, we proceed differently.

Discretization of the age $\tau$ is performed in a similar way as in [9]. We replace the semi-infinite age interval $\tau \in[0, \infty)$ by a finite interval $\left[0, \tau_{\mathrm{c}}\right]$ with $\tau_{\mathrm{c}}$ the cut-off age. This value has to be large compared to the largest relaxation time $\lambda_{\max }$ of the fluid. For the LAOS simulations in Section 7, we have used $\tau_{\mathrm{c}}=20 \lambda_{\max }$, which is a rather conservative value. The interval $\left[0, \tau_{\mathrm{c}}\right]$ is divided into $N$ subintervals $\left[\tau_{j}, \tau_{j+1}\right]$ for each $j=0, \ldots, N-1$. These subintervals are of increasing size to take advantage of the fast decaying memory function for large values of $\tau / \lambda_{i}$. For a one mode upper-convected Maxwell integral model, Hulsen et al. [9] used a stretched mesh and determined an optimal stretching factor. We found that this was not the optimal $\tau$ discretization for our multimode MSF model. To determine the mesh for the age discretization, we define a cut-off time $\tau_{\mathrm{c}, i}=20 \lambda_{i}$ for every relaxation time. For the interval $\left[0, \tau_{\mathrm{c}, 1}\right]$, we compute the $\tau$ discretization exactly as in [9]. For other intervals $\left[\tau_{\mathrm{c}, i}, \tau_{\mathrm{c}, i+1}\right]$, we use less subintervals since the region up to $\tau_{\mathrm{c}, i}$ has already been discretized based on the smaller relaxation times.

In view of the evaluation of the stress integral Eq. (1), solutions to Eq. (13) are computed in the two-point Gauss points on each subinterval. The $2 N$ Gauss points $\tau_{k}^{\mathrm{G}}$ for each $k=1, \ldots, 2 N$ define the mesh on which we compute the molecular stress function. To include the end points of the $\tau$ domain we define $\tau_{0}^{\mathrm{G}}=0$ and $\tau_{2 N+1}^{\mathrm{G}}=\tau_{\mathrm{c}}$. At each Gauss point $\tau_{k}^{\mathrm{G}}$ for $k=1, \ldots, 2 N+1$, we need to solve Eq. (13). Note that for LAOS, the Finger tensor and thus the deformation tensor is known as a function of time and $\tau$ via Eq. (12).

Integration of Eq. (13) from time $t_{i}$ to $t_{i+1}$ and over a $\tau$ interval between two Gauss points $\left[\tau_{k}^{\mathrm{G}}, \tau_{k+1}^{\mathrm{G}}\right]$ is performed using a trapezoidal rule for the time and $\tau$ direction. This results, for each $k=0, \ldots, 2 N$, in a difference equation for the molecular 
stress function at the new time level $i+1$,

$$
\begin{aligned}
f_{i+1, k+1}^{2}= & f_{i, k}^{2}+\frac{\Delta t-\Delta \tau}{\Delta t+\Delta \tau}\left(f_{i+1, k}^{2}-f_{i, k+1}^{2}\right)+\frac{1}{2} \frac{\Delta t \Delta \tau}{\Delta t+\Delta \tau} \\
& \times\left(r_{i+1, k+1}+r_{i, k+1}+r_{i+1, k}+r_{i, k}\right),
\end{aligned}
$$

where $\Delta t=t_{i+1}-t_{i}, \Delta \tau=\tau_{k+1}^{\mathrm{G}}-\tau_{k}^{\mathrm{G}}$, and $r$ denotes the righthand side of Eq. (13). All quantities $f_{i, *}^{2}$ and $r_{i, *}$ are at the previous time level and are known at the start of a new time step. The quantities $f_{i+1,0}^{2}$ and $r_{i+1,0}$ are known from the boundary condition at $\tau=0, f_{i+1,0}^{2}=1$. For the first $\tau$ interval $\left[0, \tau_{1}\right]$, only the quantities $f_{i+1,1}^{2}$ and $r_{i+1,1}$, which is a non-linear function of $f^{2}$, are unknown. To handle the non-linearity of the right-hand side, we use a predictor-corrector scheme, for which we use $f_{i, j+1}^{2}$ as a predictor. Once $f_{i+1,1}^{2}$ is known, we can apply the same procedure to obtain $f_{i+1,2}^{2}$ and so on, till we have computed $f_{i+1,2 N+1}^{2}$ at the cut-off age of the largest relaxation time.

The molecular stress function for a linear polymer melt has to remain in the range $\left(0, f_{\max }^{2}\right)$. When we use Eq. (14) in LAOS simulations, we found that $f^{2}$ can easily exceed the lower and upper bound under strongly non-linear flow conditions, particularly at large ages $\tau$ where the difference between two consecutive ages is large. This makes the method unstable. For example, once $f^{2}$ becomes negative for some value of $\tau$ it remains negative and rapidly increases in magnitude. The instability disappeared when we applied a transformation that ensures that the molecular stress function always remains between its lower and upper bound. For all our computations, we used the transformation

$h=\ln \frac{f^{2}}{f_{\max }^{2}-f^{2}}$

which maps $\left(0, f_{\max }^{2}\right)$ to $(-\infty, \infty)$. Instead of solving the differential equation for $f^{2}$, we solve the corresponding differential equation for $h$. Since $-\infty<h<\infty$, we cannot violate any constraints. After obtaining the value of $h$ at a new time level, the value of $f^{2}$ is recovered using the inverse mapping

$f^{2}=\frac{\mathrm{e}^{h} f_{\max }^{2}}{1+\mathrm{e}^{h}}$

which indeed ensures that $0<f^{2}<f_{\max }^{2}$.

In terms of time $t$ and age $\tau$, the integral for the stress, Eq. (1), becomes

$\boldsymbol{T}(t)=5 \int_{0}^{\infty} m(\tau) f^{2}(t, \tau) \boldsymbol{Q}[\boldsymbol{B}(t, \tau)] \mathrm{d} \tau$.

Once the molecular stress function is computed at the new time level, the polymer stress $\boldsymbol{T}\left(t_{i+1}\right)$ can be computed by integrating over $\tau$. On the interval $\left[0, \tau_{\mathrm{c}}\right]$, the integral is approximated by a finite sum and on $\left[\tau_{\mathrm{c}}, \infty\right)$ we assume $f^{2}(t, \tau)=f^{2}\left(t, \tau_{\mathrm{c}}\right)$ and $\boldsymbol{B}(t, \tau)=\boldsymbol{B}\left(t, \tau_{\mathrm{c}}\right)$, so that the integral can be integrated exactly. The resulting stress at the new time level is of the form

$$
\begin{aligned}
\boldsymbol{T}\left(t_{i+1}\right)= & 5 \sum_{k=1}^{2 N} w_{k} m\left(\tau_{k}^{\mathrm{G}}\right) f^{2}\left(t_{i+1}, \tau_{k}^{\mathrm{G}}\right) \boldsymbol{Q}\left[\boldsymbol{B}\left(t_{i+1}, \tau_{k}^{\mathrm{G}}\right)\right] \\
& +5 M\left(\tau_{\mathrm{c}}\right) f^{2}\left(t_{i+1}, \tau_{\mathrm{c}}\right) \boldsymbol{Q}\left[\boldsymbol{B}\left(t_{i+1}, \tau_{\mathrm{c}}\right)\right]
\end{aligned}
$$

where $w_{k}, k=1, \ldots, 2 N$ are the weights corresponding to a two-point Gauss quadrature rule and $M\left(\tau_{\mathrm{c}}\right)=$ $\sum_{i} G_{i} \exp \left(-\tau_{\mathrm{c}} / \lambda_{i}\right)$

The numerical simulation of the Doi-Edwards model in LAOS is more straightforward since no evolution equation needs to be solved for $f^{2}$. For every time step, only the stress needs to be computed using Eq. (16) with $f^{2}=1$ and the analytic solution for the Finger tensor Eq. (12). For this we use exactly the same age discretization as for the MSF model.

\section{Melt properties}

The linear spectrum of the Finathene melt that we use in all our viscoelastic simulations is the six-mode spectrum identified in [8] where it was used for LAOS simulations with the Giesekus model.

In addition to the linear spectrum, the MSF model only contains two non-linear parameters for the molecular stress function, $a_{1}$ and $a_{2}$. The parameter $a_{1}$ describes the melt rheology in nonrotational flows and its value can be obtained from fitting elongational data. Next, the value of $a_{2}$, which is relevant for rotational flows, can be determined from the shear viscosity and first normal stress difference. For the Finathene melt, however, only shear data are available. This is not a major limitation since the impact of the parameter $a_{1}$ in large amplitude oscillatory shear flows is very small as we show in Section 7. In this section, we use the value $a_{1}=0.02$ which was identified in [2] for another high-density polyethylene melt.

The second parameter $a_{2}$ is identified using steady shear viscosity data. These shear data were obtained from small amplitude oscillatory shear measurements and the Cox-Merz rule which is valid for the Finathene fluid [8]. For another highdensity polyethylene melt, the value of $a_{2}=2.3$ was identified in [2]. Using this parameter value, steady shear viscosity predictions also agree well with experimental data of the Finathene fluid as can be observed from Fig. 1. At shear rates of order unity, the experimental data are slightly overpredicted while for $\dot{\gamma}>10 \mathrm{~s}^{-1}$ a slight underprediction is apparent. Increasing the value of $a_{2}$ leads to better agreement with experimental data at shear rates around $\dot{\gamma} \approx 1 \mathrm{~s}^{-1}$. The underprediction of the data

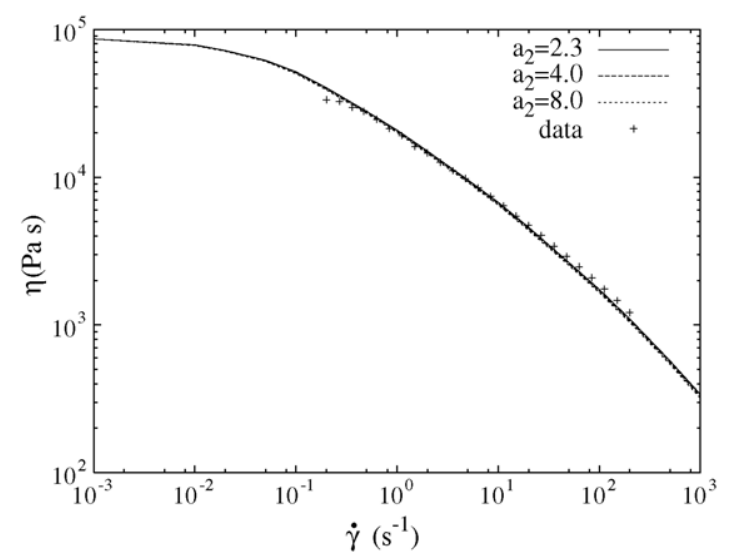

Fig. 1. Steady shear viscosity of the Finathene melt: experimental data of [8] and model predictions of the MSF model using various values of $a_{2}\left(a_{1}=0.02\right)$. 


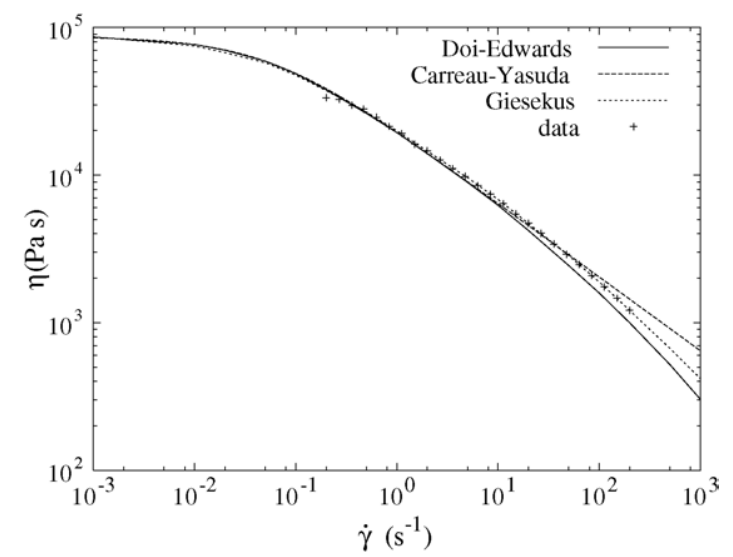

Fig. 2. Steady shear viscosity of the Finathene melt: experimental data of [8] and model predictions of the Doi-Edwards, Carreau-Yasuda, and Giesekus model.

for $\dot{\gamma}>10 \mathrm{~s}^{-1}$, however, persists and even slightly increases when $a_{2}$ is increased. We conclude from Fig. 1 that the steady shear viscosity predictions are not very sensitive to changes in $a_{2}$ and that a rather wide range of parameter values $a_{2}$ fits the data equally well. For this reason, we will consider both $a_{2}=2.3,4$, and 8 in the LAOS simulations in Section 7.

Results of the MSF model in large amplitude oscillatory shear will be compared with the basic Doi-Edwards model, the Giesekus model, and the inelastic Carreau-Yasuda model [12]. For both viscoelastic models, we use the same linear spectrum as for the MSF model. For the Giesekus model, we use in addition the non-linear parameter values identified in [8]. The viscosity of the Carreau-Yasuda model is given by

$\eta=\eta_{0}\left(1+\left[\lambda I_{2}\right]^{a}\right)^{(n-1) / a}$,

where $I_{2}$ is the second invariant of the rate-of-strain tensor $\boldsymbol{d}$. The four adjustable parameters in the model are the zero-shear viscosity $\eta_{0}$, a time constant $\lambda$, the power-law index $n$, and a numerical parameter $a$. The parameters are obtained from fitting the shear viscosity. We found a good fit using $\eta_{0}=8.66 \times 10^{4} \mathrm{~Pa} \mathrm{~s}$, $\lambda=18 \mathrm{~s}, n=0.5$, and $a=0.85$

The steady shear viscosity predictions of the Doi-Edwards and Carreau-Yasuda models are displayed in Fig. 2. Surpris- ingly, the predictions of the Doi-Edwards model, which has no adjustable parameters, agree well with the data up to moderate shear rates. At higher shear rates, $\dot{\gamma}>10 \mathrm{~s}^{-1}$, the Doi-Edwards model underpredicts the experimental data. Up to shear rates that can be reached in the LAOS experiments $\left(\dot{\gamma}<32 \mathrm{~s}^{-1}\right)$, however, differences are relatively small. In fact, the predictions of the DE model are only slightly lower than those of the MSF model with $a_{2}=8$ as can be observed by comparing Figs. 1 and 2 . The Carreau-Yasuda fit overpredicts the viscosity at large shear rates. However, for the range of shear rates in the LAOS experiments, $\dot{\gamma} \leq 32 \mathrm{~s}^{-1}$, the purely viscous model shows good agreement. For further reference, we have also included in Fig. 2 the steady shear viscosity predictions of the Giesekus model. The Giesekus model shows excellent agreement up to the largest experimental shear rate.

To analyse shear predictions for a transient flow like LAOS, it is also important to know the response in transient rheometrical flows. Fig. 3 displays the viscosity in start up of shear and start up of uniaxial elongation at various deformation rates. The differences between the results of the MSF models with different values of the non-linear parameter $a_{2}$ are small. Both $a_{2}=2.3$, 4 , and 8 show small time overshoots at moderate shear rates. The magnitudes of these overshoots and the steady viscosity slightly decrease with increasing value of $a_{2}$. For the largest value of $a_{2}=8$, the predictions lie only just above those of the Doi-Edwards model. For further reference, we also include in Fig. 3a the model predictions of the Giesekus model. There are two differences with the molecular models. First, the larger time overshoots. This becomes apparent at relatively low shear rates of $\dot{\gamma}=1$ and becomes more pronounced when the shear rate is increased. Second, the Giesekus model has a higher steady shear viscosity at high shear rates. This is in better agreement with the data in Fig. 1. For the LAOS experiments, however, such high rates cannot be achieved experimentally.

Fig. $3 \mathrm{~b}$ shows that the differences between the models is much more pronounced in start up of uniaxial extension, at significantly large values of the extension rate $\dot{\epsilon}$. The MSF model shows considerable strain hardening which increases when $a_{1}$ is increased. This behaviour is absent for the Doi-Edwards model.
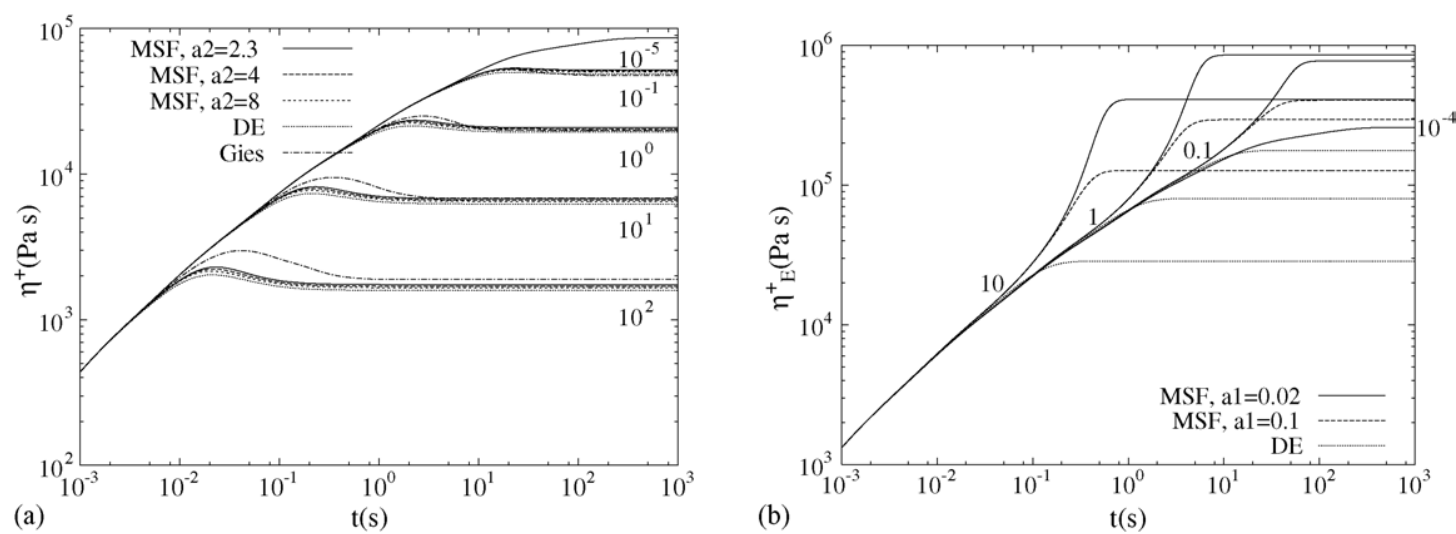

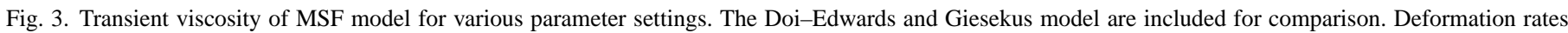

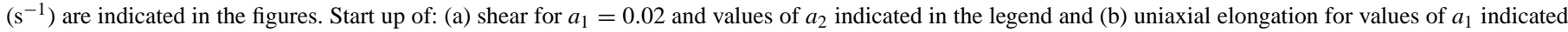
in the legend ( $a_{2}$ irrelevant). 

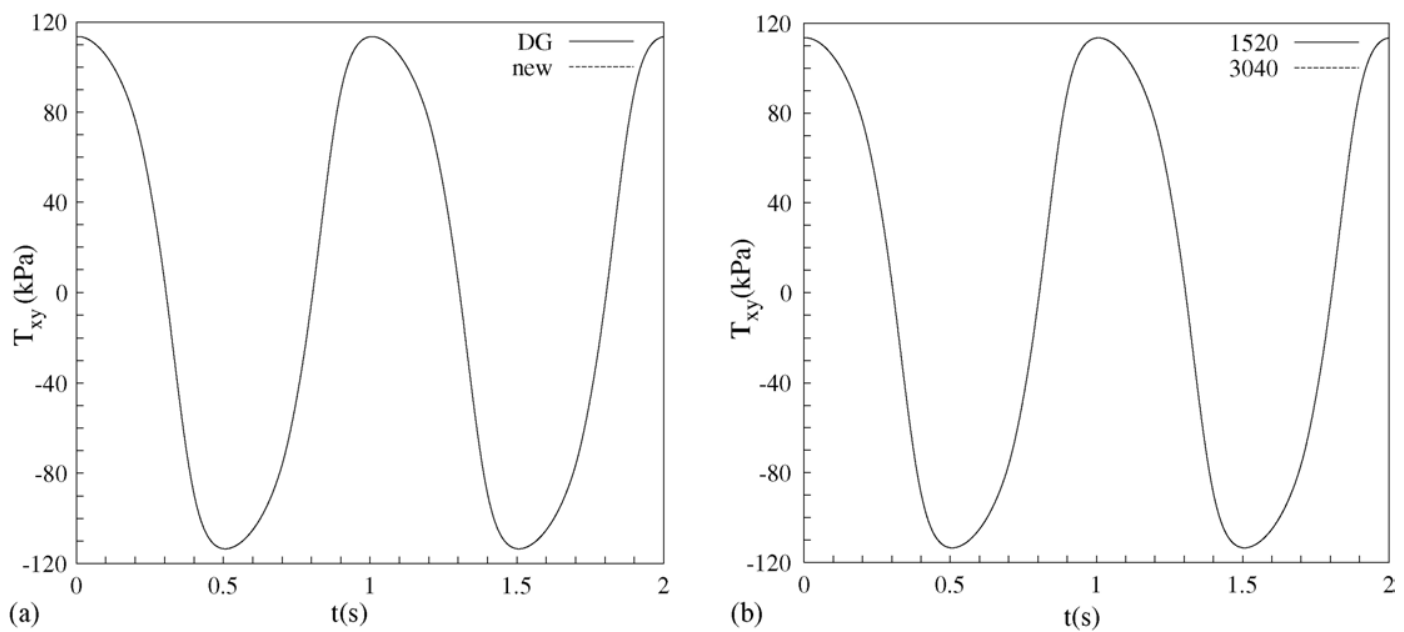

Fig. 4. Validation of the numerical technique. Comparison of: (a) new method with the DG method of [9] and (b) various $\tau$ discretizations.

\section{Validation of the numerical results}

For the validation of the numerical technique and various numerical parameters, we use the MSF model for linear polymers with parameter values $a_{1}=0.02$ and $a_{2}=2.3$. Other parameter settings, not shown in this section, gave identical results.

We first validate our numerical technique described in Section 4 , by comparing with the deformation field method using the discontinuous Galerkin method to discretise the $\tau$ direction in the equation governing the molecular stress function. This is exactly the same approach as discussed in [9] for the Finger tensor. For both techniques we take the same $\tau$ discretization using $1520 \tau$ subintervals. Fig. 4a shows the results of both techniques in LAOS with $v=1 \mathrm{~Hz}$ and $\gamma_{0}=5$ which has the highest achievable shear rate in the experiments. At the scale of the plot the methods are indistinguishable. The main difference lies in the time step employed. For the new method we have used a time step of $\Delta t=2.5 \times 10^{-4} \mathrm{~s}$ while for the DG method a time step of $\Delta t=\Delta \tau / 6$ is necessary for reasons of stability [9]. For a $\tau$ discretization using 1520 subintervals, the minimum length of a subinterval equals $\Delta \tau_{\min }=9.6 \times 10^{-6} \mathrm{~s}$. This requires a time step smaller than $\Delta t=1.6 \times 10^{-6} \mathrm{~s}$. In combination with the long time required to reach a periodic steady state, such small time steps lead to long simulation times for the DG method. The new technique, however, remains stable for much larger time steps leading to substantially smaller computation times.

Second, it needs to be checked whether the discretization in $\tau$ using $N=1520$ subintervals is sufficient to capture the steady periodic stress response. In order to verify this, we compare with a $\tau$ discretization that has twice as many subintervals, $N=$ 3040, which implies a smallest $\tau$ subinterval which is halved, $\tau_{\min }=4.8 \times 10^{-6} \mathrm{~s}$. For both computations, we used a time step of $\Delta t=2.5 \times 10^{-4} \mathrm{~s}$. The steady periodic shear stress for both $\tau$ discretizations is displayed in Fig. 4b. On the scale of the figure there are no differences between the two $\tau$ discretizations. Henceforth, we use the smallest number of subintervals, $N=$ 1520 , for all simulations with the MSF model.

A time step of $\Delta t=2.5 \times 10^{-4} \mathrm{~s}$ is sufficiently small to capture the steady periodic regime as can be observed from Fig. 5a. When the time step is halved to $\Delta t=1.25 \times 10^{-4} \mathrm{~s}$, both time steps produce identical results on the scale of the figure. For both simulations, we used $N=1520$ subintervals for the $\tau$ dis-
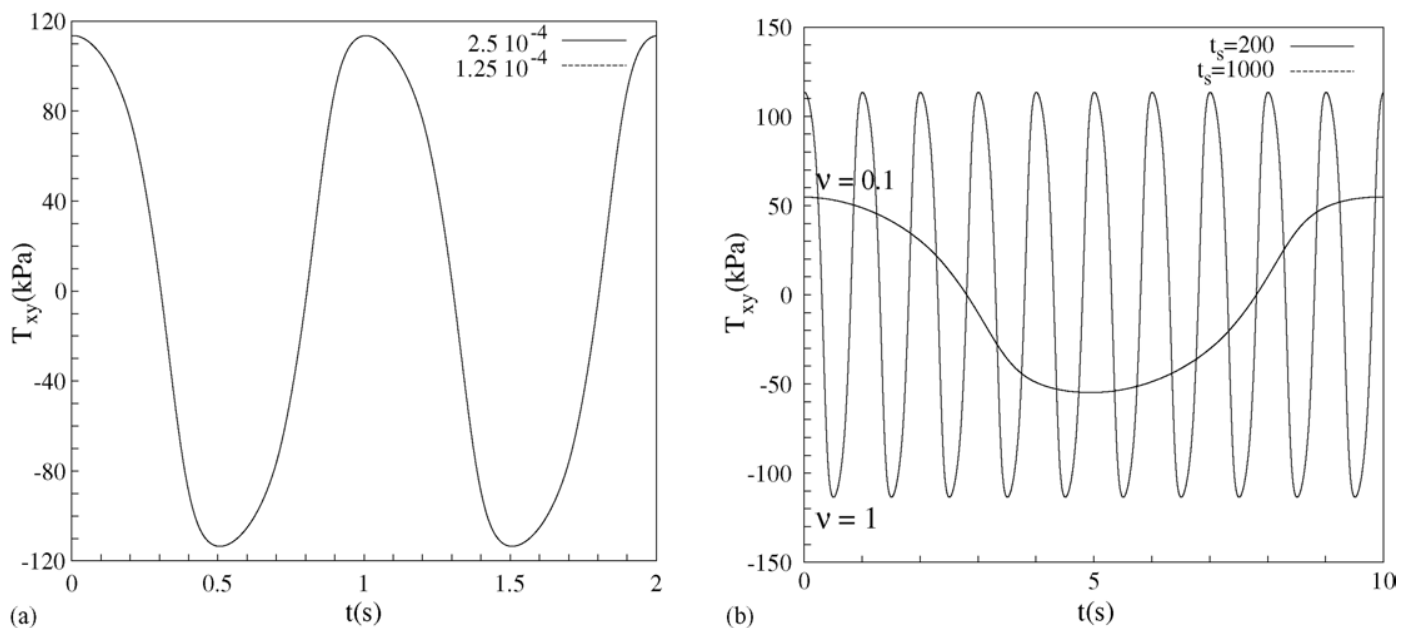

Fig. 5. Validation of sufficiently small time step (a) and sufficiently large $t_{\mathrm{s}}(\mathrm{b})$. 
cretization. Henceforth, we use $\Delta t=2.5 \times 10^{-4} \mathrm{~s}$ for all LAOS simulations.

In Section 7, LAOS simulations are performed for a time of $t_{\mathrm{s}}=200 \mathrm{~s}$. Fig. $5 \mathrm{~b}$ compares shear stresses using $t_{\mathrm{s}}=200$ and $1000 \mathrm{~s}$ for the highest experimentally achievable amplitudes at a low $(v=0.1 \mathrm{~Hz})$ and a high frequency $(v=1 \mathrm{~Hz})$. For both calculations, we used $\Delta t=2.5 \times 10^{-4} \mathrm{~s}$ and $N=1520$. We conclude from Fig. $5 \mathrm{~b}$ that $t_{\mathrm{s}}=200 \mathrm{~s}$ is sufficiently large to reach the steady periodic regime. This value of $t_{\mathrm{s}}$ is a rather conservative choice. The stress maxima only differ $0.1 \%$ from the steady periodic value after 3 and 15 cycles for $v=0.1$ and $1 \mathrm{~Hz}$, respectively.

\section{Large amplitude oscillatory shear results}

The MSF theory uses the strain measure based on the independent alignment approximation. In view of the large number of time steps that have to be performed, it is advantageous to use the computationally much more efficient Currie approximation instead. This is a good approximation in steady and start up of shear for the Doi-Edwards model [10]. It is, however, a priori not clear how well both strain measures correspond in large amplitude oscillatory shear flows of an MSF fluid. For this we compared the steady periodic shear stress at a low $(v=0.1 \mathrm{~Hz})$ and high frequency $(v=1 \mathrm{~Hz})$ for the two highest amplitudes $\gamma_{0}$ that are experimentally achievable for each frequency. As can be seen from Fig. 6, the flow curves of the Currie and independent alignment approximation agree very well. Only near the minima and maxima we observe that the Currie approximation slightly overpredicts the independent alignment approximation. This is, however, negligible compared to the differences between the various model predictions and the experimental data as we discuss shortly. The approximation is equally good for the other two frequencies, $v=0.3$ and $3 \mathrm{~Hz}$, which are not shown in Fig. 6. We conclude from Fig. 6 that in large amplitude oscillatory shear simulations of an MSF fluid, the Currie approximation is a very good approximation to the independent alignment approximation of the Doi-Edwards strain mea-

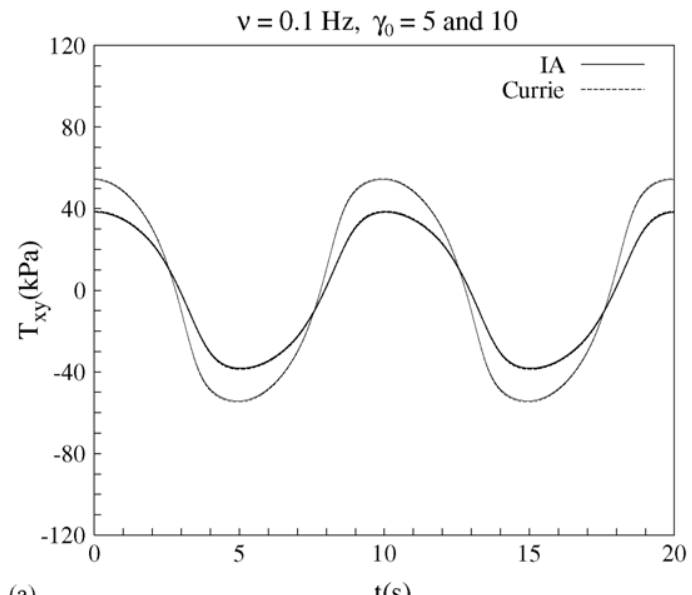

(a)

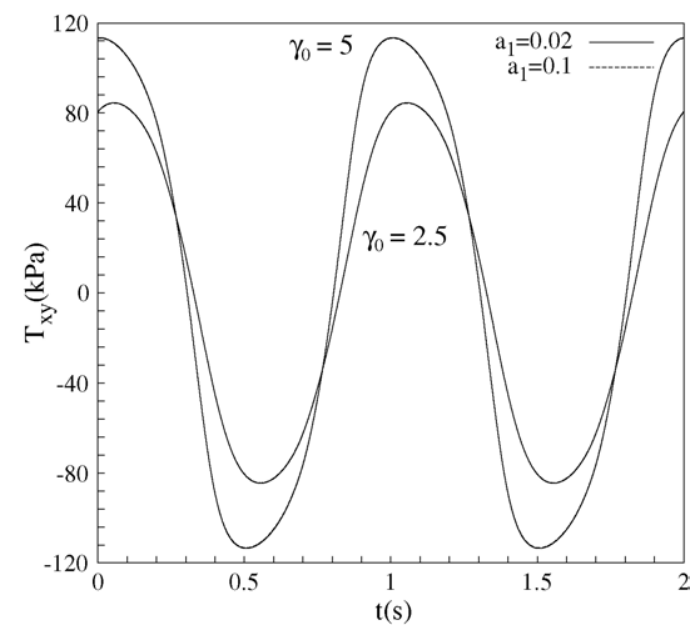

Fig. 7. Steady periodic shear stress at $v=1 \mathrm{~Hz}$ for the MSF model using $a_{2}=$ 2.3 and values of $a_{1}$ indicated in the legend.

sure. Henceforth, we use the Currie approximation in all LAOS simulations.

To accurately determine the parameter $a_{1}$ of the MSF model for a polymeric fluid, extensional data are required. For the Finathene melt, however, only shear data are available. Therefore, we first compare the periodic steady shear response for two realistic values of $a_{1}$. For the first value, we take $a_{1}=0.02\left(f_{\max }^{2}=51\right)$ as identified in [2] for another high-density polyethylene melt. The linear polymers considered in [13] have a lower $f_{\max }^{2}$. For the second value, we take $f_{\max }^{2}=11$ which corresponds to $a_{1}=0.1$. Fig. 7 shows the impact of $a_{1}$ on the periodic steady shear response for $v=1 \mathrm{~Hz}$ at the two highest experimentally achievable amplitudes $\gamma_{0}=2.5$ and 5. At the scale of the figure, both parameter values of $a_{1}$ result in identical model predictions. We conclude that the only relevant MSF model parameter for large amplitude oscillatory shear flow of linear polymers is $a_{2}$ and henceforth we use $a_{1}=0.02$ for all simulations.

The LAOS experiments have been performed at various frequencies and amplitudes. At low values of $v$ and $\gamma_{0}$, the flow does not deviate much from low amplitude oscillatory shear. It is

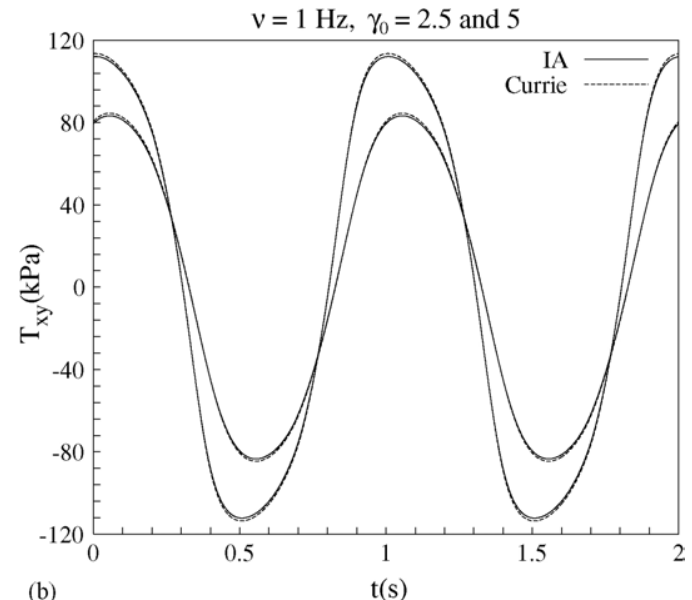

(b)

$\mathrm{t}(\mathrm{s})$

Fig. 6. Comparison of the steady periodic shear stress prediction of the MSF model using the Currie and independent alignment approximation at amplitudes $\gamma_{0}$ indicated in the figures: (a) $v=0.1 \mathrm{~Hz}$ and (b) $v=1 \mathrm{~Hz}$. 

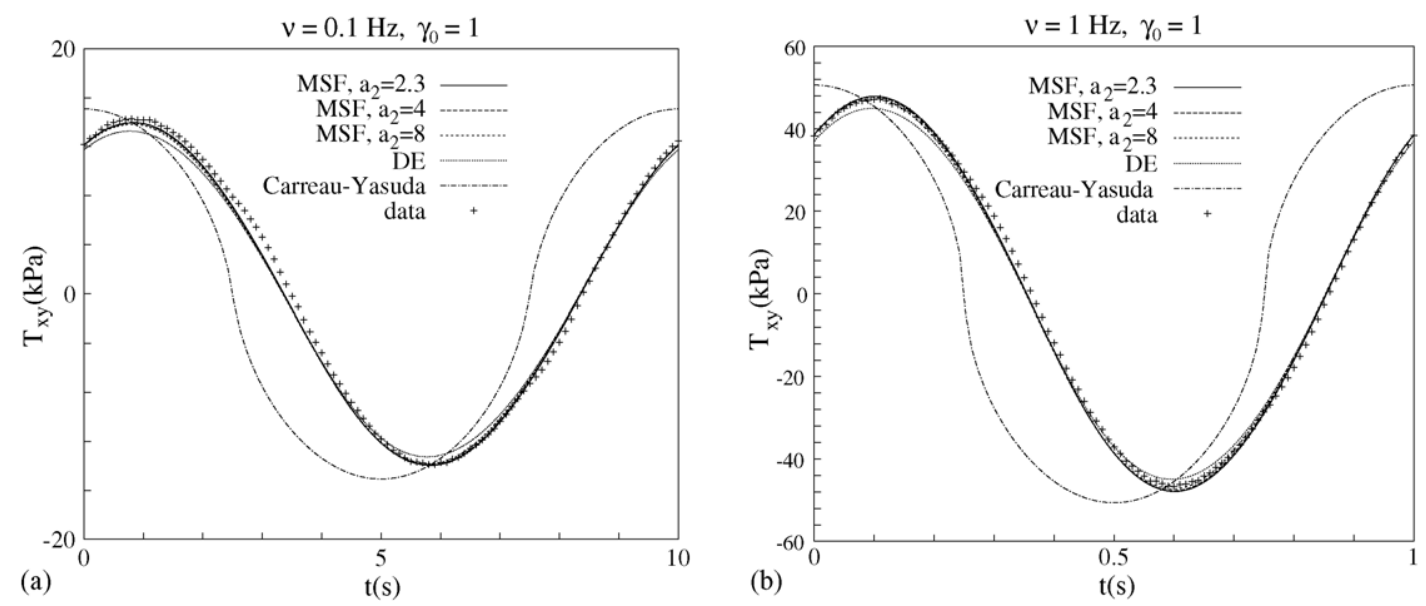

Fig. 8. Periodic shear stress at moderate shear rates predicted by the viscous and viscoelastic models together with the Finathene data. Frequencies and strains indicated in the figures.

therefore not surprising that all viscoelastic models predict similar results that do not differ much from the experimental data. As an example, we display for $\gamma_{0}=1$ the steady periodic response for $v=0.1$ and $1 \mathrm{~Hz}$ in Fig. 8. All viscoelastic models considered predict the correct phase shift of the periodic shear stress. The MSF model, for all parameter values of $a_{2}$ considered, also correctly predict the amplitude. The Doi-Edwards model, however, clearly underpredicts the amplitude in the mildly non-linear regime. To investigate the impact of viscoelasticity, we also display results of an inelastic model possessing only shear-thinning behaviour. This model is only able to predict the correct amplitude and fails to predict the phase shift. At $v=1 \mathrm{~Hz}$ and $\gamma_{0}=1$, which corresponds to a maximum shear rate of $\dot{\gamma} \approx 6.3 \mathrm{~s}^{-1}$, the inelastic model also significantly overpredicts the amplitude of the periodic shear stress signal.

At larger values of $\gamma_{0}$, differences between the various parameter settings in the MSF model and the Doi-Edwards model become more apparent. In Fig. 9, we display for each frequency the steady periodic shear stress for the two largest experimentally achievable values of $\gamma_{0}$. At the lowest strains, the Doi-Edwards model consistently underpredicts the experimental data, while the MSF predictions still show good agreement for all values of $a_{2}$ considered, particularly $a_{2}=4$. For the larger $\gamma_{0}$ (and thus larger shear rates) the Doi-Edwards predictions are again fairly close to the experimental data. Surprisingly, the results are in better agreement with the experiments than most of the predictions of the MSF model which has two extra parameters to capture the non-linear behavior. The best MSF parameter for the largest strains seems to be $a_{2}=8$. The parameter values $a_{2}=4$ and particularly $a_{2}=2.3$ overpredict the shear response. This is most apparent at $v=1 \mathrm{~Hz}$ and $\gamma_{0}=5$ which has the highest maximum shear rate. We note in passing that the Giesekus model also overpredicts the experimental signal at more non-linear flow conditions [8]. At $v=1 \mathrm{~Hz}$ and $\gamma_{0}=5$, the predicted shear stress amplitude is, for example, $117 \mathrm{kPa}$ for the Giesekus model, while the largest amplitude that occurs for the MSF model is $113.5 \mathrm{kPa}$ for $a_{2}=2.3$. At this amplitude and frequency, the amplitude of the experimental signal $106 \mathrm{kPa}$ is best predicted by $a_{2}=8$ which gives an amplitude of
$106.8 \mathrm{kPa}$. The Doi-Edwards model slightly underpredicts the amplitude, $101.8 \mathrm{kPa}$. Similar trends are visible at the lower frequencies $v=0.1$ and $0.3 \mathrm{~Hz}$. At $v=3 \mathrm{~Hz}$, no data are available at high enough $\gamma_{0}$ to confirm the counter intuitive result that the Doi-Edwards model gives better predictions at large strains (large shear rates). It is remarkable that at corresponding values of the shear rate, the steady shear viscosity data in Fig. 1 are better predicted by the MSF and Giesekus model than by the Doi-Edwards model which shows a stronger underprediction of the shear viscosity at high shear rates. We also observe that the LAOS simulations are more sensitive to changes in the parameter $a_{2}$ than steady shear flow at corresponding shear rates. This indicates that large oscillatory shear might be a better flow to determine the non-linear parameters that are important for shearing flows.

The Lissajous plot for a frequency $v=1 \mathrm{~Hz}$ and all experimentally available strain amplitudes are depicted in Fig. 10. All viscoelastic models predict the deviation from an ellipsoidal shape that is characteristic for the non-linear response at large strains. Quantitative differences are observed at the largest strain $\gamma_{0}=5$ for the Giesekus model and the MSF model, particularly with $a_{2}=2.3$ and to a lesser extent with $a_{2}=4$. At this strain, predictions of the MSF model with $a_{2}=8$ and Doi-Edwards model are in good agreement with the experimental data. At lower strains, however, some discrepancies are noticeable for the Doi-Edwards model. For all viscoelastic models, deviations are in accordance with the over and underprediction of the amplitude of the periodic shear stress signal as a function of time observed in Fig. 9.

For the first normal stress difference $N_{1}$, no experimental data are available. For completeness, we have included the model predictions at experimentally achievable strains for $v=1 \mathrm{~Hz}$ in Fig. 11. The periodic $N_{1}$ response has a period that is half of the corresponding shear stress signal and has a non-zero average. All models predict the same phase shift for all strains and nearly the same minimum value of $N_{1}$. The difference between the models lie in the maxima of the amplitudes. Similar to the periodic shear stress, the Doi-Edwards model predicts the lowest and the MSF model with smallest value of $a_{2}$ the 

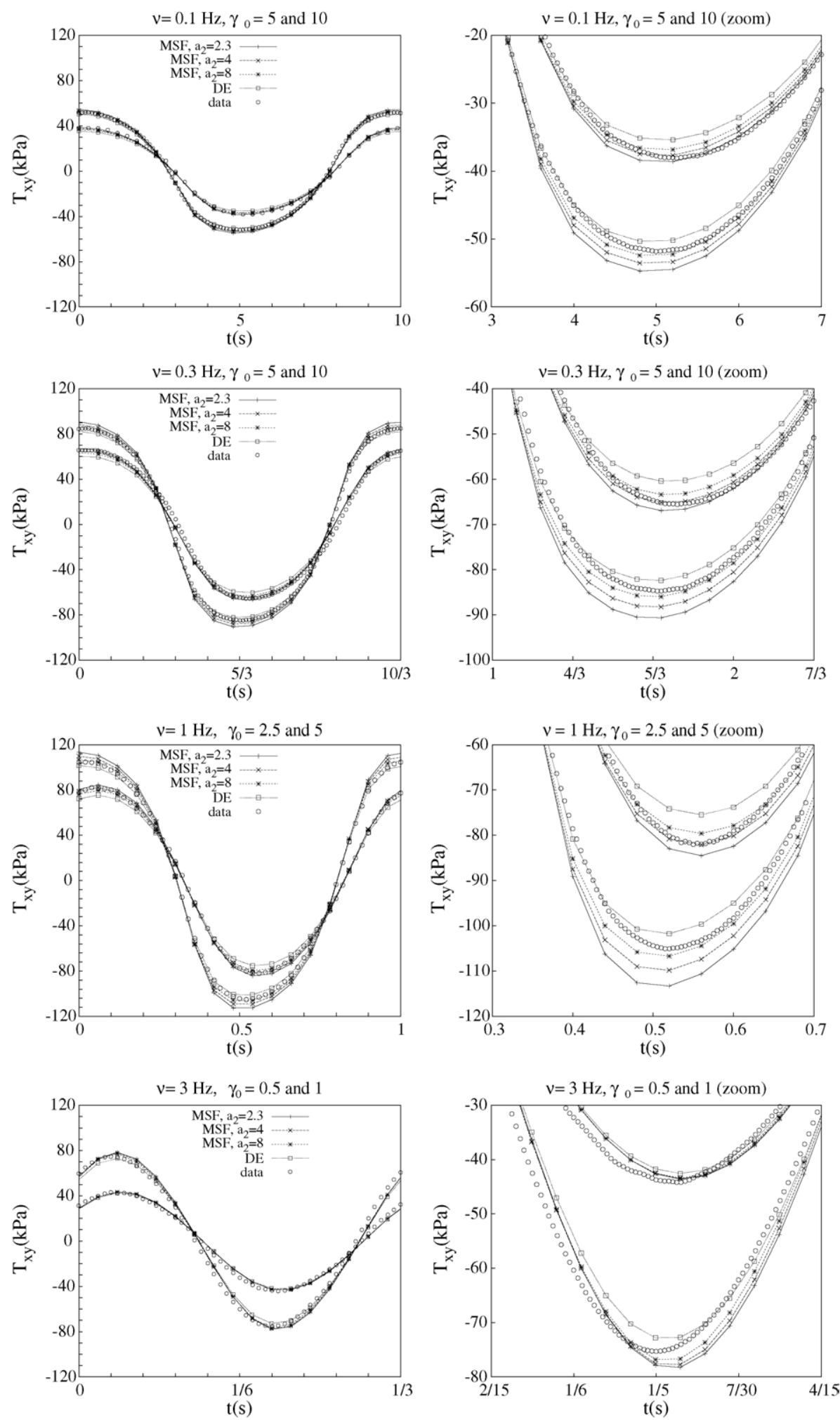

Fig. 9. Comparison of MSF model using values of $a_{2}$ as in the legends and Doi-Edwards model with periodic shear stress data for Finathene melt [8]. Left column: one period; right column: zoom around minimum.

largest amplitude. Different magnitudes of the amplitudes become already apparent at relatively low strains of $\gamma=1$ where the shear stress predictions are still very similar, particularly for the various values of $a_{2}$ of the MSF model (Fig. 8). The periodic normal stress, however, can clearly be distinguished and differ- ences between the models continue to grow when the strain is increased. It would be interesting to compare these results with experimental data to establish whether the Doi-Edwards model also better predicts the first normal stress difference at high strains. 

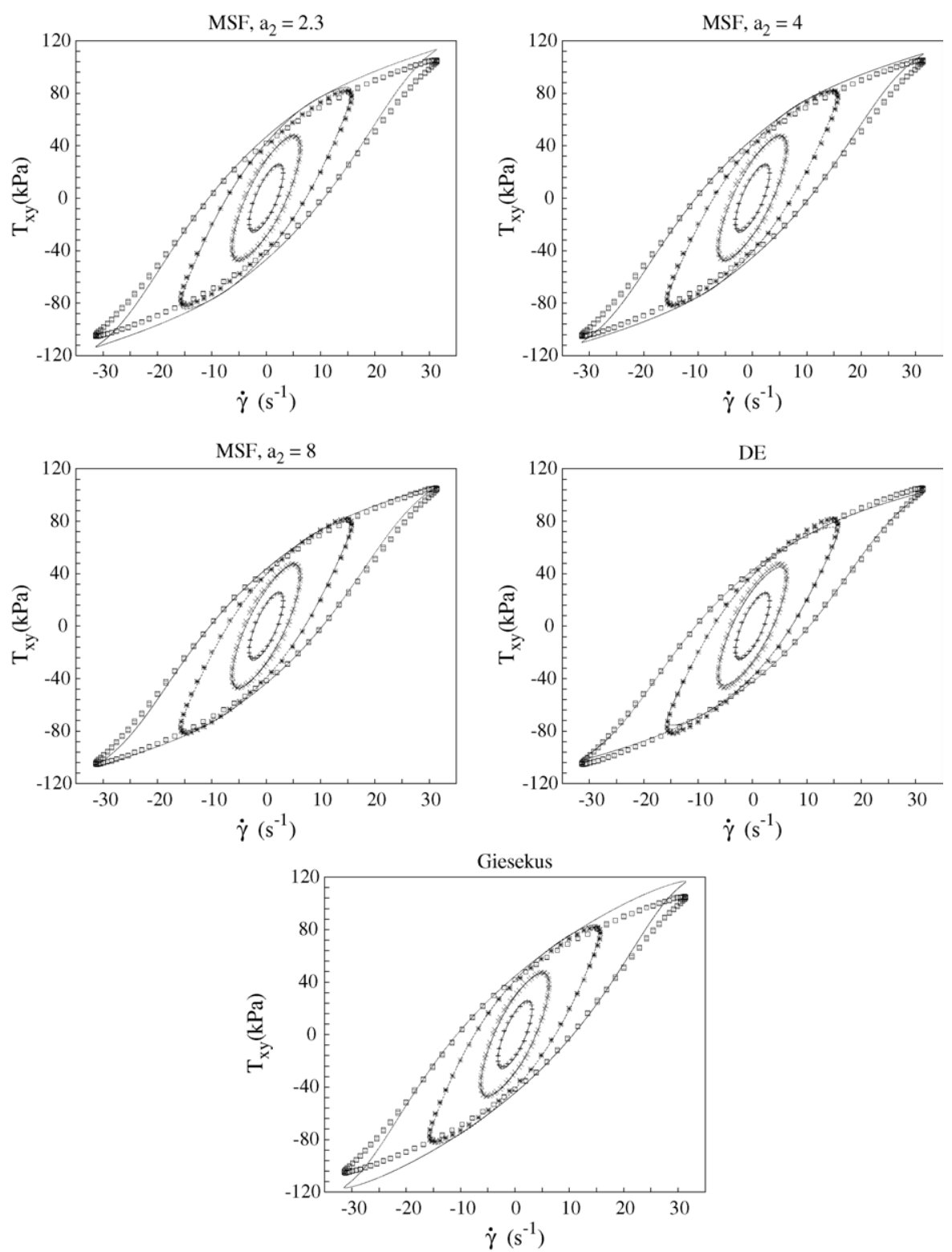

Fig. 10. Lissajous plots for $v=1 \mathrm{~Hz}$ for Finathene data of [8] and model predictions. Strain amplitudes $\gamma_{0}$ are $0.5,1,2.5$, and 5 (from innermost to outermost loops).
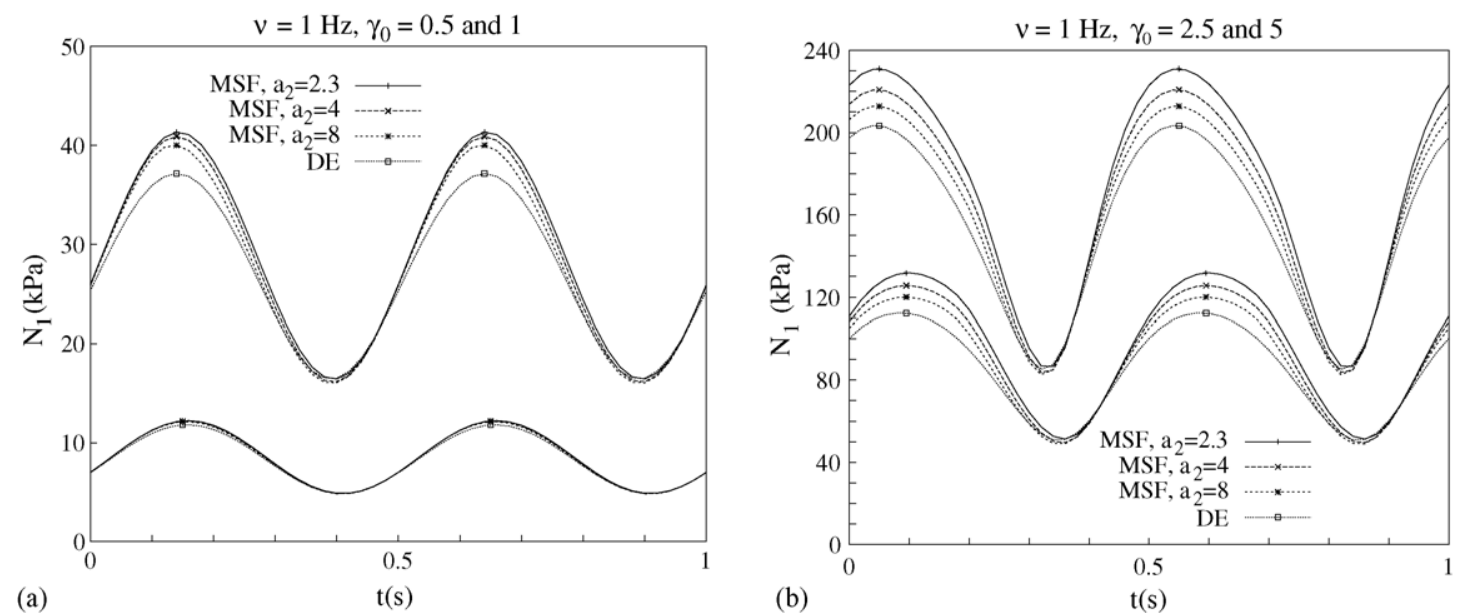

Fig. 11. Comparison of $N_{1}$ predictions for the MSF model using values of $a_{2}$ as indicated in the legends and the Doi-Edwards model. 
Table 1

Amplitudes $A_{n}$ and $B_{n}$ of the odd harmonics $(\mathrm{kPa})$ for $v=0.1 \mathrm{~Hz}$ (Comparison of experimental data and MSF results using various values of $a_{2}$. )

\begin{tabular}{|c|c|c|c|c|c|c|c|c|c|}
\hline \multirow[t]{2}{*}{$\gamma_{0}$} & \multirow[t]{2}{*}{$n$} & \multicolumn{4}{|l|}{$A_{n}(\mathrm{kPa})$} & \multicolumn{4}{|l|}{$B_{n}(\mathrm{kPa})$} \\
\hline & & Experimental & $a_{2}=2.3$ & $a_{2}=4$ & $a_{2}=8$ & Experimental & $a_{2}=2.3$ & $a_{2}=4$ & $a_{2}=8$ \\
\hline \multirow[t]{4}{*}{5} & 1 & 10.9 & 9.07 & 8.69 & 8.39 & 38.8 & 39.7 & 38.9 & 38.1 \\
\hline & 3 & -2.84 & -3.32 & -3.25 & -3.13 & -0.34 & -0.598 & -0.761 & -0.847 \\
\hline & 5 & 0.259 & 0.146 & 0.199 & 0.231 & -0.534 & -0.499 & -0.499 & -0.487 \\
\hline & 7 & 0.027 & 0.139 & 0.159 & 0.160 & 0.116 & 0.001 & 0.029 & 0.068 \\
\hline \multirow[t]{4}{*}{10} & 1 & 8.44 & 7.41 & 7.14 & 6.94 & 55.3 & 58.7 & 57.5 & 56.4 \\
\hline & 3 & -4.15 & -4.92 & -4.70 & -4.51 & -3.43 & -3.75 & -3.83 & -3.85 \\
\hline & 5 & 1.38 & 1.48 & 1.47 & 1.43 & -0.219 & -0.505 & -0.391 & -0.311 \\
\hline & 7 & -0.295 & -0.030 & -0.073 & -0.096 & 0.37 & 0.435 & 0.431 & 0.419 \\
\hline
\end{tabular}

Differences between the model predictions are better quantified in the frequency domain than in the time domain. Under more non-linear flow conditions, the higher odd harmonics become more significant. The even harmonics should vanish except for some numerical noise. For the following computations, the order of magnitude of the even harmonics was at least 6 orders lower than that of the largest odd harmonics. We focus on the two highest experimentally achievable amplitudes at a low frequency of $v=0.1 \mathrm{~Hz}$ and a high frequency of $v=1 \mathrm{~Hz}$.

We first consider the two highest experimentally achievable strains at the lowest frequency $v=0.1 \mathrm{~Hz}$. Table 1 shows the Fourier coefficients $A_{n}$ and $B_{n}$ for the MSF model using various values of $a_{2}$. At this low frequency the response in phase with the shear rate is dominating and becomes more dominant when the strain is increased. This is correctly predicted by the MSF model for all parameter values considered. Furthermore, the MSF model predicts the signs of all Fourier coefficients correctly. There are only small quantitative differences. At the low strain $\gamma_{0}=5$, the low harmonics agree best for $a_{2}=4$, particularly the dominating amplitude $B_{1}$ which corresponds to the lost work per cycle [4]. At the high strain $a_{2}=8$ gives the best agreement for the dominating amplitude $B_{1}$. This explains the better agreement of $a_{2}=4$ at $\gamma_{0}=5$ and of $a_{2}=8$ at $\gamma_{0}=10$ in Fig. 9.

The amplitudes of the first four non-zero harmonics for the Doi-Edwards, Carreau-Yasuda, and Giesekus model are tabulated in Table 2. The amplitudes of the Doi-Edwards model closely follow the MSF results with $a_{2}=8$, with the exception of $B_{1}$ which is somewhat lower. At $\gamma_{0}=5$, the underprediction of the amplitude of the experimental shear stress signal in Fig.
9 is caused by an underprediction of the first harmonics $A_{1}$ and $B_{1}$. The higher harmonics of the Doi-Edwards model are, however, in good agreement with the experimental data, while the Giesekus model predicts the signs of some higher harmonics incorrectly. It is remarkable that at the larger strain of $\gamma_{0}=10$, the differences with the experimental $A_{1}$ and $B_{1}$ are considerably smaller, resulting in a slightly better agreement with the experimental data than the best MSF model at this $\gamma_{0}$. Furthermore, the higher harmonics of the Doi-Edwards model are in excellent agreement with the experimental data while the Giesekus model shows again much larger deviations and predicts some signs incorrectly.

At the frequency $v=0.1 \mathrm{~Hz}$ and at the strain values in Table 2, the Carreau-Yasuda model is still able to predict correctly the coefficient $B_{1}$, i.e. the lost work per cycle. The amplitudes $B_{3}, B_{5}$, and $B_{7}$, however, are off by a factor $2-10$ and these might have the wrong sign. Furthermore, the inelastic model only predicts non-zero values for the coefficients $B_{n}$ and is therefore incapable to predict the phase shift in the shear stress response.

At the frequency $v=1 \mathrm{~Hz}$, the response in phase with the strain is much larger than for the low frequency of $v=0.1 \mathrm{~Hz}$. This is correctly predicted by the MSF model. The deviations between the experiments and the MSF results are caused by an overprediction of $B_{1}$, as can be observed from Table 3. For the parameter value $a_{2}=2.3$, the value of $B_{1}$ is overpredicted by at least $5 \%$ and deviations become larger when the strain increases. For larger values of $a_{2}$, the deviations from the experimentally obtained $B_{1}$ become smaller. Particularly, $a_{2}=8$ agrees well with the experimental data. For some other amplitudes like $A_{3}$ and $B_{3}$, however, we then find larger differ-

Table 2

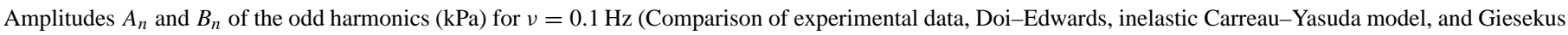
model. Data for the Giesekus model are from [8].)

\begin{tabular}{|c|c|c|c|c|c|c|c|c|c|}
\hline \multirow[t]{2}{*}{$\gamma_{0}$} & \multirow[t]{2}{*}{$n$} & \multicolumn{4}{|l|}{$A_{n}(\mathrm{kPa})$} & \multicolumn{4}{|l|}{$B_{n}(\mathrm{kPa})$} \\
\hline & & Experimental & $\mathrm{DE}$ & CY & G & Experimental & $\mathrm{DE}$ & CY & G \\
\hline \multirow[t]{4}{*}{5} & 1 & 10.9 & 8.06 & - & 8.19 & 38.8 & 36.6 & 39.3 & 39.2 \\
\hline & 3 & -2.84 & -3.02 & - & -2.90 & -0.34 & -0.799 & -5.29 & -0.163 \\
\hline & 5 & 0.259 & 0.297 & - & -0.124 & -0.534 & -0.499 & 2.30 & -0.704 \\
\hline & 7 & 0.027 & 0.103 & - & 0.151 & 0.116 & 0.106 & -1.32 & -0.062 \\
\hline \multirow[t]{4}{*}{10} & 1 & 8.44 & 6.70 & - & 6.51 & 55.3 & 54.1 & 56.2 & 59.4 \\
\hline & 3 & -4.15 & -4.34 & - & -4.34 & -3.43 & -3.71 & -7.76 & -3.27 \\
\hline & 5 & 1.38 & 1.42 & - & 1.20 & -0.219 & -0.284 & 3.43 & -1.11 \\
\hline & 7 & -0.295 & -0.152 & - & 0.388 & 0.37 & 0.411 & -2.01 & 0.419 \\
\hline
\end{tabular}


Table 3

Amplitudes $A_{n}$ and $B_{n}$ of the odd harmonics (kPa) for $v=1 \mathrm{~Hz}$ (Comparison of experimental data and MSF results using various values of $a_{2}$.)

\begin{tabular}{|c|c|c|c|c|c|c|c|c|c|}
\hline \multirow[t]{2}{*}{$\gamma_{0}$} & \multirow[t]{2}{*}{$n$} & \multicolumn{4}{|l|}{$A_{n}(\mathrm{kPa})$} & \multicolumn{4}{|l|}{$B_{n}(\mathrm{kPa})$} \\
\hline & & Experimental & $a_{2}=2.3$ & $a_{2}=4$ & $a_{2}=8$ & Experimental & $a_{2}=2.3$ & $a_{2}=4$ & $a_{2}=8$ \\
\hline \multirow[t]{3}{*}{2.5} & 1 & 38.0 & 38.6 & 37.0 & 35.6 & 74.6 & 79.2 & 77.7 & 75.7 \\
\hline & 5 & -0.04 & -0.458 & -0.550 & -0.576 & -0.512 & -0.082 & -0.178 & -0.346 \\
\hline & 7 & 0.098 & 0.100 & 0.128 & 0.161 & 0.296 & -0.083 & -0.073 & -0.029 \\
\hline \multirow[t]{2}{*}{5} & 1 & 33.1 & 34.5 & 33.0 & 31.9 & 108 & 117 & 114 & 111 \\
\hline & 3 & -9.66 & -12.4 & -12.1 & -11.7 & -1.23 & -1.79 & -2.43 & -2.75 \\
\hline
\end{tabular}

ences with the experiments. These amplitudes are off by at least $25 \%$.

Table 4 shows the corresponding results for the DoiEdwards, Giesekus, and Carreau-Yasuda model. In this more non-linear regime, the purely viscous model is not capable to predict any of the amplitudes correctly. Also, $B_{1}$ is now overpredicted considerably. The Doi-Edwards model underpredicts both $A_{1}$ and $B_{1}$ at the lower strain $\gamma_{0}=2.5$. For this strain,

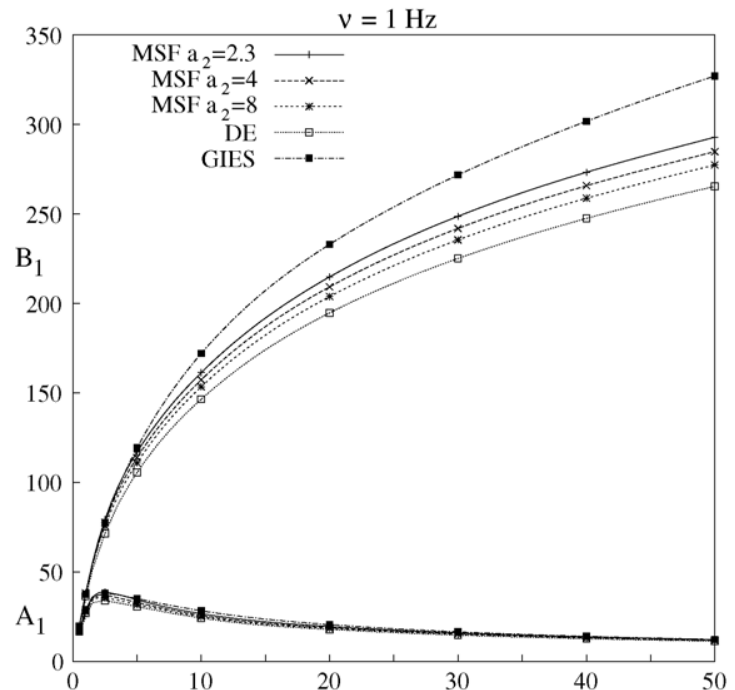

(a)

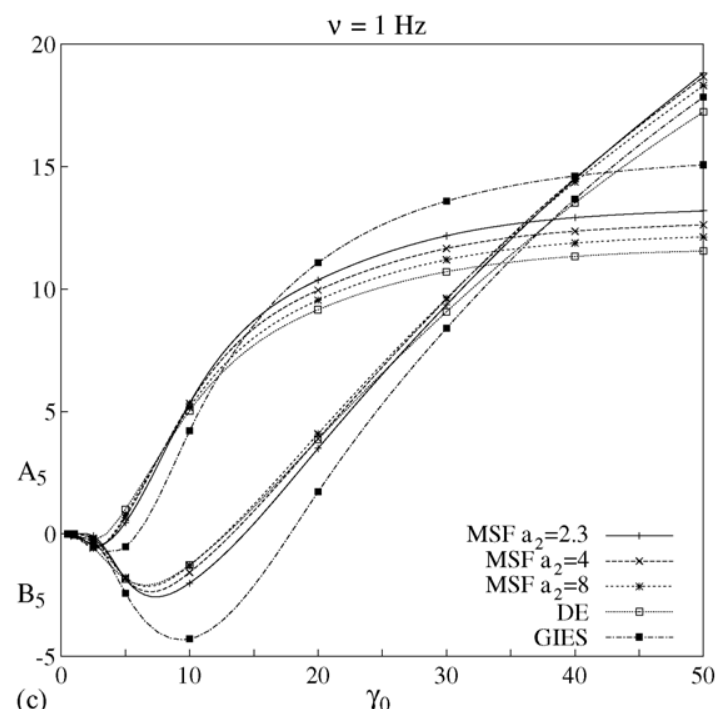

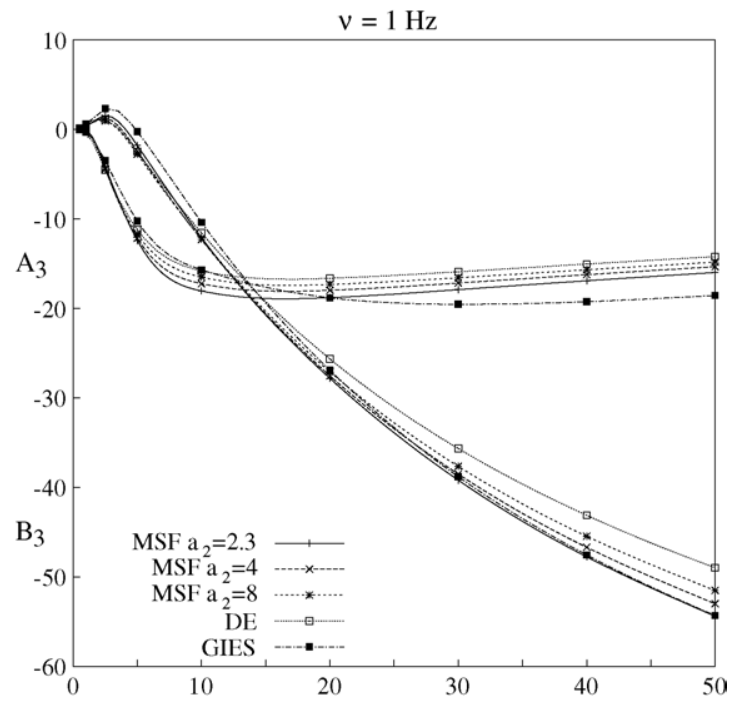

(b)

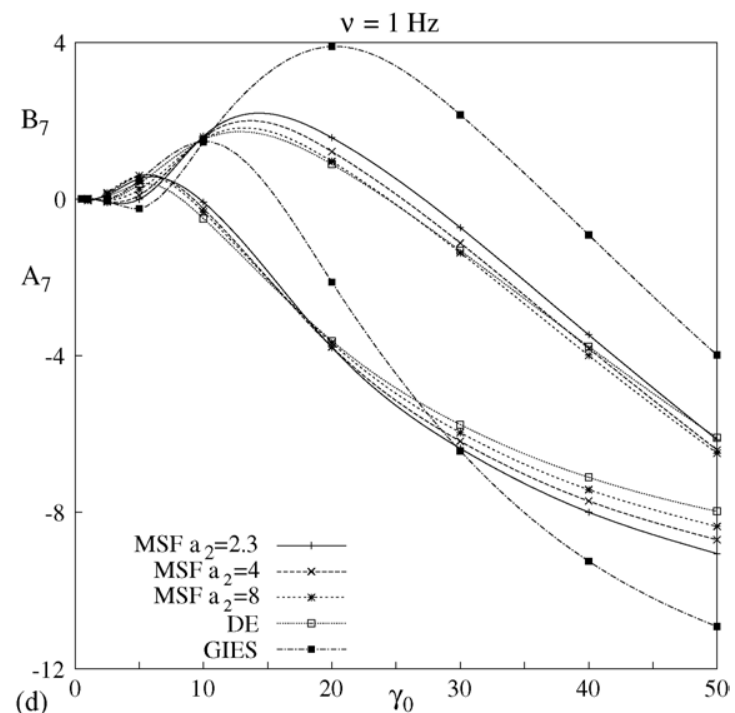

Fig. 12. (a-d) Amplitudes $A_{n}$ and $B_{n}$ of the odd harmonics ( $\mathrm{kPa}$ ) outside the experimental window for MSF model using values of $a_{2}$ as in the legends. The Doi-Edwards and Giesekus model are included for comparison. 
Table 4

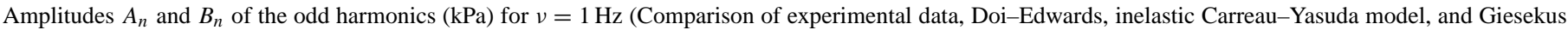
model. Data for the Giesekus model are from [8].)

\begin{tabular}{|c|c|c|c|c|c|c|c|c|c|}
\hline \multirow[t]{2}{*}{$\gamma_{0}$} & \multirow[t]{2}{*}{$n$} & \multicolumn{4}{|l|}{$A_{n}(\mathrm{kPa})$} & \multicolumn{4}{|l|}{$B_{n}(\mathrm{kPa})$} \\
\hline & & Experimental & $\mathrm{DE}$ & $\mathrm{CY}$ & G & Experimental & $\mathrm{DE}$ & $\mathrm{CY}$ & G \\
\hline \multirow[t]{4}{*}{2.5} & 1 & 38.0 & 34.1 & - & 38.2 & 74.6 & 71.6 & 89.5 & 77.8 \\
\hline & 3 & -2.92 & -4.52 & - & -3.45 & 2.40 & 1.14 & -12.6 & 2.31 \\
\hline & 5 & -0.04 & -0.274 & - & -0.474 & -0.512 & -0.538 & 5.65 & -0.227 \\
\hline & 7 & 0.098 & 0.063 & - & -0.008 & 0.296 & -0.025 & -3.35 & -0.059 \\
\hline \multirow[t]{4}{*}{5} & 1 & 33.1 & 30.7 & - & 35.1 & 108 & 106 & 127 & 119 \\
\hline & 3 & -9.66 & -11.2 & - & -10.2 & -1.23 & -2.46 & -18.0 & -0.211 \\
\hline & 5 & 0.817 & 0.996 & - & -0.492 & -1.62 & -1.85 & 8.11 & -2.42 \\
\hline & 7 & 0.075 & 0.391 & - & 0.492 & 0.412 & 0.385 & 4.83 & -0.242 \\
\hline
\end{tabular}

the amplitudes of the low harmonics are better predicted by the Giesekus and MSF model. The higher harmonics, which arise due to non-linear effects, are again much better predicted by the Doi-Edwards than by the Giesekus model. For the more non-linear regime at the higher strain, the Doi-Edwards model does not only show better agreement with experimental data for the higher harmonics. Also, the dominating term $B_{1}$ is much better predicted than the Giesekus model does. This confirms the counter intuitive result found for the lower frequency of $v=0.1 \mathrm{~Hz}$ that the Doi-Edwards model, which has no nonlinear parameters and underpredicts the steady shear viscosities at high rates, is more accurate in the more non-linear regimes.

Fig. 12 shows the model predictions of the MSF and DoiEdwards model outside the experimental window. To compare the model predictions with a macroscopic stress model, results for the Giesekus model have been included as well. All models predict the same trends at large strains, for example, the same sign is predicted for all Fourier coefficients $B_{n}$ and $A_{n}$ at large strains. The dominating amplitude is $B_{1}$, while the magnitude of $A_{1}$ decreases at large strains and reaches a plateau. For the higher harmonics, all amplitudes $B_{n}$ in phase with the shear seem to reach a higher magnitude at large strains than the corresponding amplitudes $A_{n}$ that are in phase with the strain. The Fourier coefficient $B_{n}$ seems to continue to increase in magnitude for a much longer range of strains than the corresponding $A_{n}$, which reaches a plateau value. For the two lowest harmonics, the plateau is reached at medium strains of 10. For the higher harmonics much higher strains are necessary. Quantitatively, however, there are differences between the models. Only for $A_{1}$, all models predict the same values. For all other Fourier coefficients, the Doi-Edwards model predicts the lowest magnitudes. At large strains, the amplitudes are considerably lower than those of the MSF model with $a_{2}=8$. For the MSF model, the magnitudes of the odd harmonics increase when the parameter $a_{2}$ is decreased. The magnitudes predicted by the MSF model with $a_{2}=2.3$ are, however, still considerably lower than those predicted by the Giesekus model. Particularly for the dominant amplitude $B_{1}$ the molecular models predict significantly lower magnitudes at large strains. This is consistent with the smaller time overshoots for these models in the transient shear viscosity in Fig. 3a. Whether the trends at large strains are correct and whether the Doi-Edwards predictions are still more accurate than those of the non-linear models remains to be established.

\section{Concluding remarks}

We have evaluated the rheometrical response of the integral molecular stress function model in large amplitude oscillatory shear. For reasons of efficiency, we modified the deformation field method to avoid the restrictive time step constraint resulting from the discontinuous Galerkin method used in [9]. The new technique is both accurate and efficient for the LAOS computations we performed.

In LAOS, the MSF model only has one relevant material parameter to describe the non-linear material response. By fitting this parameter to steady shear data of a linear high-density polyethylene melt, the MSF model is able to predict qualitatively and quantitatively the response in large amplitude oscillatory shear. Up to medium strains, results agree very well with experimental data and are comparable with those of a six-mode Giesekus fluid, having six parameters to describe the non-linear material response. At medium strains, the non-linear response becomes important. As expected, the introduction of the molecular stress function results in better predictions than the basic Doi-Edwards theory which has no material parameters to describe the non-linear response. Although correctly predicting the phase shift, the DE model underpredicts the amplitude of the experimental signal. This is caused by an underprediction of the amplitude of the most dominant odd harmonics.

At the highest experimentally achievable strains, both the MSF and Giesekus model overpredict the amplitude of the periodic shear stress, although this is more significant for the Giesekus model. At these strains, however, it is the Doi-Edwards model that shows excellent agreement with the experimental data. In the time domain it only slightly underpredicts the amplitude of the periodic shear stress. In the frequency domain the correspondence of both the dominant harmonic and the higher odd harmonics is striking. We recall that the DE model does not have any non-linear parameters and underpredicts the steady shear stress at high shear rates. This is a remarkable and unexpected result indeed. Whether this trend continues at higher strains remains to be established. Since the Doi-Edwards model is well known to underpredict experimental data in start up of shear and steady shear flows, this also raises the question whether the experimental data are somewhat inaccurate at high strains. Further experimental results are clearly needed to confirm our counter intuitive findings at high strains. 


\section{Acknowledgements}

We wish to thank Henri Burhin and Christian Bailly for making the Finathene data available to us. We also thank Manfred Wagner for fruitful discussions. This work is supported by the programme Action de Recherche Concertée, Communauté Française de Belgique.

\section{References}

[1] M. Doi, S.F. Edwards, The Theory of Polymer Dynamics, Clarendon Press, Oxford, 1986.

[2] M.H. Wagner, P. Rubio, H. Bastian, The molecular stress function model for polydisperse polymer melts with dissipative convective constraint release, J. Rheol. 45 (2001) 1387-1412.

[3] W. Philippoff, Vibrational measurements with large amplitudes, Trans. Soc. Rheol. 10 (1966) 317-334.

[4] A.J. Giacomin, J.M. Dealy, Using large-amplitude oscillatory shear, in: A.A. Collyer, D.W. Clegg (Eds.), Rheological Measurements, second ed., Kluwer Academic Publishers, Dordrecht, 1998, pp. 327-356.

[5] M. Wilhelm, D. Maring, H.W. Spiess, Fourier-transform rheology, Rheol. Acta 37 (1998) 399-405.
[6] M. Wilhelm, P. Reinheimer, M. Ortseifer, High sensitivity Fouriertransform rheology, Rheol. Acta 38 (1999) 349-356.

[7] D. van Dusschoten, M. Wilhelm, H.W. Spiess, Two-dimensional Fourier transform rheology, J. Rheol. 45 (2001) 1319-1339.

[8] B. Debbaut, H. Burhin, Large amplitude oscillatory shear and Fourier transform rheology for a high-density polyethylene: experiments and numerical simulation, J. Rheol. 46 (2002) 1155-1176.

[9] M.A. Hulsen, E.A.J.F. Peters, B.H.A.A. van den Brule, A new approach to the deformation fields method for solving complex flows using integral constitutive equations, J. Non-Newt. Fluid Mech. 98 (2001) 201221.

[10] P.K. Currie, Constitutive equations for polymer melts predicted by the Doi-Edwards and Curtiss-Bird kinetic theory models, J. Non-Newt. Fluid Mech. 11 (1982) 53-68.

[11] M.H. Wagner, M. Yamaguchi, M. Takahashi, Quantitative assesssment of strain hardening of low-density polyethylene melts by the molecular stress function model, J. Rheol. 47 (2003) 779-793.

[12] R.B. Bird, R.C. Armstrong, O. Hassager, Dynamics of Polymeric Liquids, vol. 1, second ed., John Wiley, New York, 1987.

[13] M.H. Wagner, H. Bastian, A. Bernnat, S. Kurzbeck, C.K. Chai, Determination of elongational viscosity of polymer melts by RME and Rheotens experiments, Rheol. Acta 41 (2002) 316-325. 